Research Article

\title{
Sound Velocity Estimation of Seabed Sediment Based on Parametric Array Sonar
}

\author{
Jingxin Ma $\mathbb{D}^{1,2,3}$ Haisen Li, ${ }^{1,2,3}$ Jianjun Zhu $\mathbb{D}^{1,2,3}$ and Baowei Chen ${ }^{1,2,3}$ \\ ${ }^{1}$ Acoustic Science and Technology Laboratory, Harbin Engineering University, Harbin 150001, China \\ ${ }^{2}$ Key Laboratory of Marine Information Acquisition and Security (Harbin Engineering University), \\ Ministry of Industry and Information Technology, Harbin 150001, China \\ ${ }^{3}$ College of Underwater Acoustic Engineering, Harbin Engineering University, Harbin 150001, China
}

Correspondence should be addressed to Jianjun Zhu; zhujianjun_heu@hrbeu.edu.cn

Received 5 July 2020; Revised 24 July 2020; Accepted 27 July 2020; Published 14 August 2020

Academic Editor: Ivan Giorgio

Copyright (c) 2020 Jingxin Ma et al. This is an open access article distributed under the Creative Commons Attribution License, which permits unrestricted use, distribution, and reproduction in any medium, provided the original work is properly cited.

\begin{abstract}
Backscattered sound waves of seabed sediments are important information carriers in seafloor detection and acoustic characteristic parameters inversion. Most of the existing methods for estimating geoacoustic parameters are based on multiangle seabed backscattered signal processing and suitable for flat seafloor conditions with uniform sediment thickness. This usually deviates from the real field conditions and affects the accuracy of parameter estimation. In this paper, the sound ray propagation theory is studied and analysed under the condition of sloping seabed and uneven sediment thickness. Based on the phased parameter array sonar system, a method of acoustic parameters estimation of the sediment under inclined seabed conditions is proposed. The simulation results show that the new method shows good adaptability to different inclination angles of the seabed and solves the problem of accuracy of acoustic parameter estimation of the inclined seabed sediments. The model will greatly reduce the seafloor topography requirements in the sediment acoustic parameter inversion, such as velocity, layer thickness, and acoustic impedance.
\end{abstract}

\section{Introduction}

Geoacoustic parameters of submarine sediments are the basis of shallow sea communications, seabed resource detection, and other seabed scientific research studies [1-4]. In the traditional seabed sediment detection, the problem of the coupling between sediment depth and sound velocity cannot be solved [5]. In actual detection, sub-bottom profiler systems generally use empirical sound velocity to calculate the sediment layer thickness. This method will certainly affect the detection accuracy to a certain extent [6]. In order to solve this problem, the method of acoustic parameters acquisition of seabed sediment in the measured area must be effective.

At present, most methods of acoustic parameter inversion are based on the underwater acoustic methods, such as the matching field inversion method [7-9], the seabed reflection loss inversion method $[10,11]$, and the high- resolution local seabed inversion method. Matching field inversion can estimate the geoacoustic parameters in a large area, but it can only reflect the average value of the water and seafloor spatial variation environment, lacking the accuracy of local inversion parameters [12]. The Schock [13] inversion method using the complex Biot model based on the vertical bottom reflection loss can invert the local sound velocity at high resolution, but the inversion method has a complicated calculation process. The high-resolution local seabed inversion method adopts multisound source transmission and vertical array reception in different places to complete the measurement of geoacoustic parameters in the short distance area, but the inversion efficiency is low and the project is more difficult to achieve [14]. The multiangle backscatter method is based on the common point arrangement of the transceiver; this method uses the time-delay information of echoes in different directions to measure the sound velocity of the deposited layer, and the calculation method is simple 
[15]. The flat seafloor model for inversion of sound velocity based on multiangle seabed backscatter has been well proved in theory and experiments [16-19]; however, this model is sensitive to the inclination of the seabed and cannot be applied to inclined seabed conditions. Therefore, this model cannot currently be used to measure the sound velocity of actual seabed sediments.

In this paper, an inclined seabed model based on the multiangle seabed backscatter signal is proposed. Under this assumption, there is no fluctuation in the seabed terrain within the measurement range and the sedimentary layer is isotropic. Based on the linear acoustics theory, the geometric relationship between the wave propagation paths of the upper and lower sediment surfaces received at different angles is analysed. An equation on the sound velocity of the sedimentary layer is constructed and finally solved by accurate time-delay information acquisition of the echo signals.

\section{Descriptions of the Problem}

2.1. Inclined Sediment Model. The multiangle backscattering geoacoustic parameter estimation method proposed in this article is a method for calculating seabed acoustic parameters based on phased parametric arrays to receive echo acoustic signals from the upper and lower interfaces of sediment layers at different angles. The geometric schematic diagram of the acoustic parameter estimation for the inclined submarine sedimentary layer using the phased parametric array is shown in Figure 1 (the sedimentary layer is assumed to be one layer). The sedimentary layer is isotropic in a detection area, and the boundary is straight, the oblique angle of the inclined seabed is $\theta_{0}$, and the angle between the bottom of the sedimentary layer and the seabed is $\theta_{1}$. The measuring point seabed depth $h_{i}$ can be directly measured by the primary frequency sound wave radiated by the parameter array, and the seawater sound velocity $c_{0}$ can also be directly obtained by expert measurement equipment. The thickness and sound velocity of the sediment are $H_{i}$ and $c_{s}$, respectively. The parametric array can transmit primary frequency sound beams and the corresponding difference frequency sound beams in different directions by phased control technology.

2.2. Multiangle Sound Propagation Model. Under the theory of ray acoustics, when sound waves propagate in different media, there will be an acoustic refraction effect. When the phased parametric array radiates a detection signal at angle $\varphi_{i}$, the distance $h_{i}$ from the seabed footprint to the surface of the parametric array (as shown in Figure 1) is

$$
h_{i}=\frac{1}{2} t_{i} c_{0} \cos \left(\alpha_{i}+\theta_{0}\right) \text {, }
$$

and the distance $d_{i}$ from the footprint on the lower surface of the sediment to the seabed footprint is

$$
d_{i}=\frac{1}{2}\left(T_{i}-t_{i}\right) c_{s} \sin \beta_{i}
$$

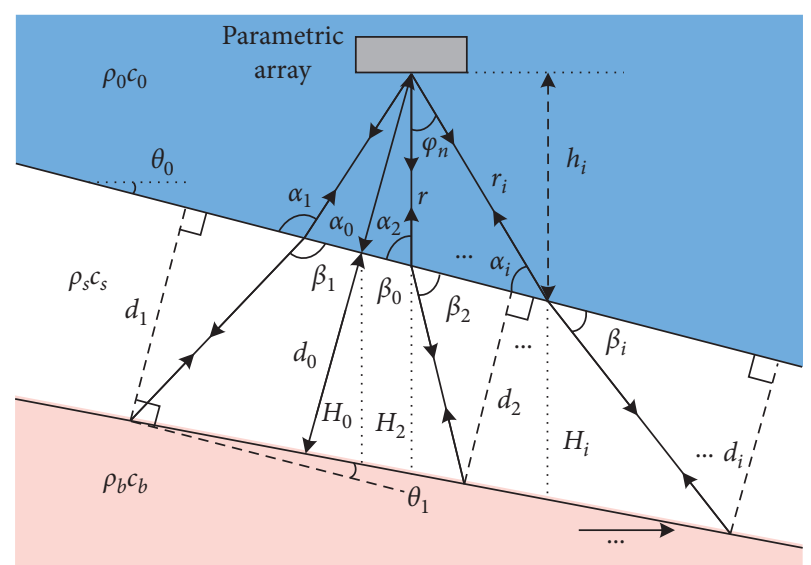

Figure 1: Geometric diagram of the sound propagation path under inclined seabed condition.

where $\alpha_{i}=\pi / 2-\theta_{0}-\varphi_{i}, i=1,2,3, \ldots, N$, is the number of sound beams radiated by the parametric array at a certain phased control angle $\varphi_{i}$ and $N$ is the total number of beams. $t_{i}$ and $T_{i}$ are the time delay of the echo signal scattered from the upper and lower surface of the sediment, respectively. $\alpha_{i}$ and $\beta_{i}$ are the glancing angle and the refraction angle of the sound beam in the sediment layer, respectively, and they are subject to Snell's law as follows:

$$
\frac{\cos \alpha_{i}}{\cos \beta_{i}}=\frac{c_{0}}{c_{s}} .
$$

When measuring the oblique angle of the seabed, the sound beams at different phase control angles should be used. According to the triangle sine theorem, there is a geometric relation as

$$
\frac{r_{i}}{\sin \alpha_{j}}=\frac{r_{j}}{\sin \alpha_{i}},
$$

where $r_{i}=t_{i} c_{0} / 2$ is the sound path from the parametric array to the upper surface of the sediment, $i, j=1,2,3, \ldots$ and $i \neq j$.

The oblique angle of the upper surface of the sediment (seabed) is

$$
\tan \left(\theta_{0}+\varphi_{j}\right)=\cot \left(\varphi_{i}-\varphi_{j}\right)-\frac{T_{j}-t_{j}}{T_{i}-t_{i}} \frac{1}{\sin \left(\varphi_{i}-\varphi_{j}\right)} .
$$

According to the geometric relationship, the inclined angle $\theta_{1}$ between the upper surface and the lower surface of the sediment can be calculated by

$$
\tan \theta_{1}=\frac{d_{0}-d_{i}}{d_{i} \cot \beta_{i}+h_{i}\left(\cos \alpha_{i} / \cos \theta_{0}\right)},
$$

where $d_{0}$ is the distance from the position of the lower surface to the upper surface when the sound beam enters the seabed vertically.

Introducing equations (1) and (2) into equation (6), we can obtain 


$$
\tan \theta_{1}=\frac{\left(T_{0}-t_{0}\right)-\left(T_{i}-t_{i}\right) \sin \beta_{i}}{\left(T_{i}-t_{i}\right) c_{s} \cos \beta_{i}+t_{i} c_{0} \cos \alpha_{i}} c_{s} .
$$

\section{Sound Velocity Solution}

It can be seen from equations (1)-(2) and (7) that the accurate thickness of the sediment is related to the sound velocity between the layers of sediment. Therefore, when the actual sound velocity in the sediment is obtained, the stratum structure of the sediment can be accurately detected. By changing the form of equation (7), the binary equations for calculating the sediment sound velocity $c_{s}$ and the angle $\theta_{1}$ using different directions can be obtained as

$$
\begin{aligned}
& {\left[\left(T_{i}-t_{i}\right) c_{s} \cos \beta_{i}+t_{i} c_{0} \cos \alpha_{i}\right] \tan \theta_{1}} \\
& \quad+\left(T_{i}-t_{i}\right) c_{s} \sin \beta_{i}-\left(T_{0}-t_{0}\right) c_{s}=0 .
\end{aligned}
$$

In this paper, the least squares problem of equation (8) is solved using the Gauss-Newton iteration algorithm [20]. Introducing equation (3) into equation (8), the equation (8) can be constructed as

$$
\begin{aligned}
f_{j}\left(x_{1}, x_{2}\right)= & {\left[\left(T_{i}-t_{i}\right) x_{1}^{2}+t_{i}\right] \cos \alpha_{i} x_{2} } \\
& +\left(T_{i}-t_{i}\right) x_{1} \sin \beta_{i}-\left(T_{0}-t_{0}\right) x_{1},
\end{aligned}
$$

where $\sin \beta_{i}=\sqrt{1-x_{1}^{2} \cos ^{2} \alpha_{i}}, \cos \beta_{i}=x_{1} \cos \alpha_{i}$, and $\left(x_{1}, x_{2}\right)=$ $\left(c_{s} / c_{0}, \tan \theta_{1}\right)$.

The first-order Taylor expansion of the binary function $f_{j}\left(x_{1}, x_{2}\right)$ at $\left(x_{1}^{0}, x_{2}^{0}\right)$ is

$$
f_{i}=f_{i}\left(x_{1}^{0}, x_{2}^{0}\right)+\sum_{k=1}^{2}\left(x_{k}-x_{k}^{0}\right) \frac{\partial}{\partial x_{k}} f_{i}\left(x_{1}^{0}, x_{2}^{0}\right)+o\left(x_{k}-x_{k}^{0}\right),
$$

where

$$
\begin{aligned}
& \frac{\partial f_{i}}{\partial x_{1}}=\left(2 x_{1} x_{2} \cos \alpha_{i}+\frac{1-2 x_{1}^{2} \cos ^{2} \alpha_{i}}{\sqrt{1-x_{1}^{2} \cos ^{2} \alpha_{i}}}\right)\left(T_{i}-t_{i}\right)-\left(T_{0}-t_{0}\right), \\
& \frac{\partial f_{i}}{\partial x_{2}}=\left[\left(T_{i}-t_{i}\right) x_{1}^{2}+t_{i}\right] \cos \alpha_{i} .
\end{aligned}
$$

Expressing function arguments in vector form $x^{0}=\left(x_{1}^{0}, x_{2}^{0}\right)$, equation (10) can be transformed into the following vector form:

$$
f\left(x^{0}\right)+J\left(x^{0}\right)\left(x-x^{0}\right)=0,
$$

where

$$
J=J\left(x_{1}, x_{2}\right)=\left[\begin{array}{llll}
\frac{\partial f_{1}}{\partial x_{1}} & \frac{\partial f_{2}}{\partial x_{1}} & \cdots & \frac{\partial f_{n}}{\partial x_{1}} \\
\frac{\partial f_{1}}{\partial x_{2}} & \frac{\partial f_{2}}{\partial x_{2}} & \cdots & \frac{\partial f_{n}}{\partial x_{2}}
\end{array}\right]^{\mathrm{T}} .
$$

When $n>2$, equation (12) is an overdetermined equation, and its least squares solution is

$$
x^{1}=x^{0}-J^{-1}\left(x^{0}\right) f\left(x^{0}\right),
$$

where $J^{-1}$ is the generalized inverse matrix, $x^{0}$ is the initial value of the equation iteration, and the solution of the equation obtained by multiple iterations is

$$
x^{k+1}=x^{k}-J^{-1}\left(x^{k}\right) f\left(x^{k}\right) .
$$

\section{Numerical Calculation and Discussion}

The depth of the seabed and the thickness of the sedimentary layer below the parametric array are $h=3$ and $H=4$, and the sound velocities of the seawater and the sedimentary layer are $c_{0}=1500$ and $c_{s}=2000$, respectively. The parametric array radiates a series of phase-controlled sound beams from $-20^{\circ}$ to $20^{\circ}$ in $0.5^{\circ}$ steps to complete the scanning of the seabed. By using the inclined seabed model, the direction of the sound beam and echo-delay information can be obtained directly. The angle of inclination $\theta_{0}$ of the seabed is estimated by the measurement in equation (5). Therefore, the echo delays of the vertical detection beam on the upper and lower surfaces of the sediments $t_{i}$ and $T_{i}$ can be determined from a series of detection beams according to the current seabed tilt angle. Equation (15) is used to solve the sound velocity of the sediment layer. The initial iteration condition is $x^{0}=(1700 /$ $1500,0)$.

4.1. Model Performance Analysis. In order to analyse the accuracy of sound speed inversion under the inclined seabed model and the flat seabed model, the sound speed inversion results calculated based on multiangle echo signals under different conditions were analysed. As shown in Figure 2(a), the calculation results show that the sound velocity is prone to large-scale drift when using the flat seabed model, and especially, the results change dramatically according to the seafloor inclined angle and the incident angle of sound beam. As a comparison, under the same conditions, using the method proposed in this paper, the results of sound velocity inversion are given in Figure 2(b). The results show that compared with the traditional flat seabed model, this inclined seabed model shows good adaptability to the more complex actual seabed conditions, such as the changed sediment thickness and seabed with different oblique angles.

The sound velocity in the sediment and the angle between the upper and lower surface of the sediment are calculated by the Gauss-Newton iterative method under different conditions. In order to analyse the convergence rate and accuracy of Equation (15), the relationship between sound speed iteration and the convergence rate and the relationship between angle iteration and the convergence rate are shown in Figure 3. The calculation results show that after five iterations, the results are close to the true values. The effectiveness of this method is verified.

4.2. Sediment Thickness Correction. Due to the refraction effect of sound rays, the propagation path of sound in the sediment does not correspond with the depth of the 


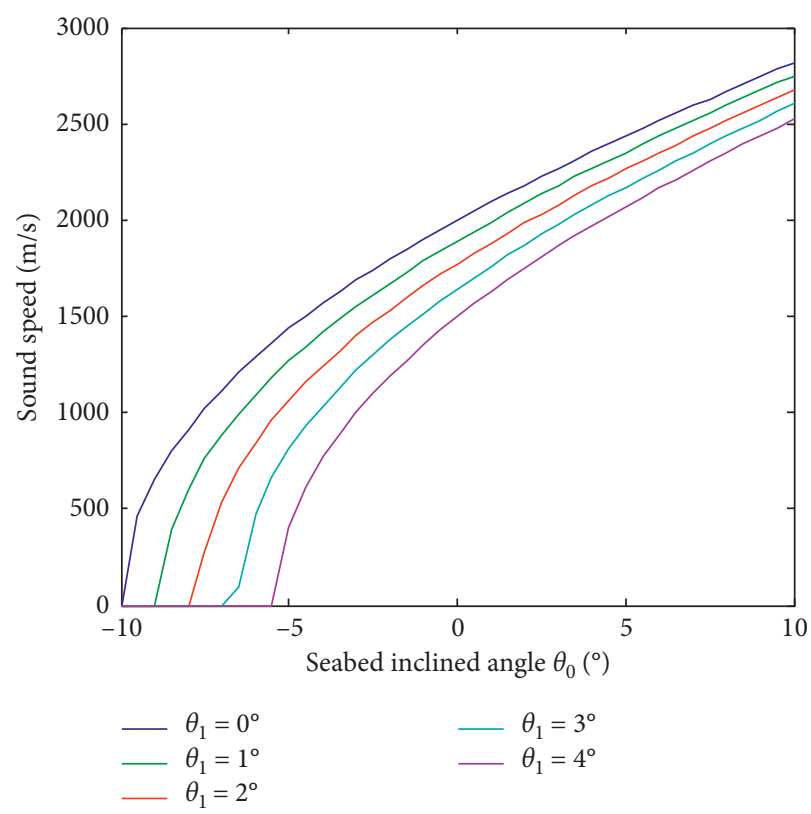

(a)

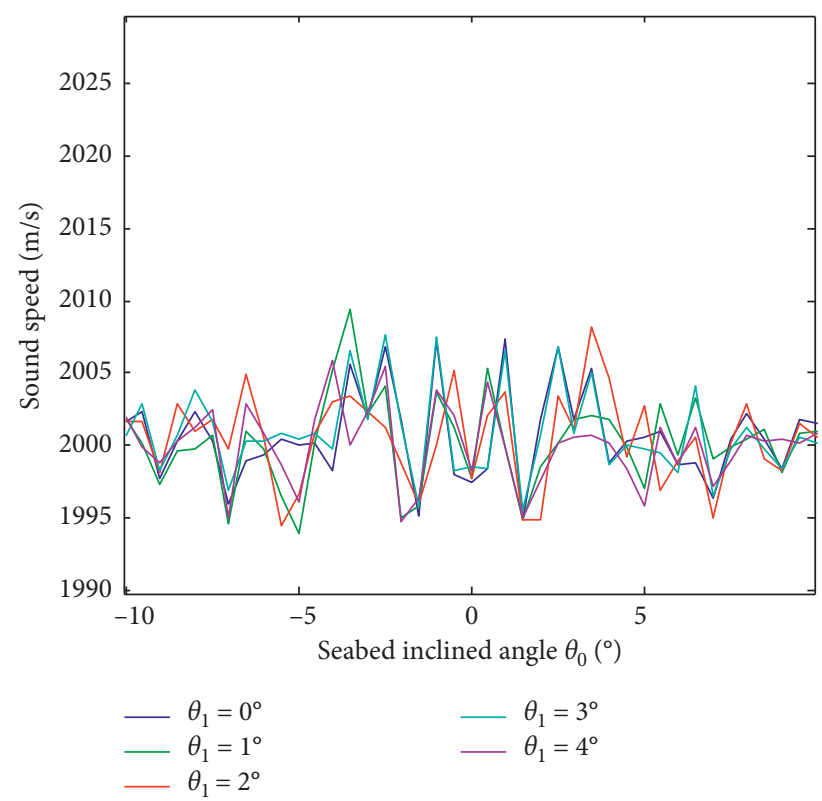

(b)

FIGURE 2: Sediment velocity estimation under different seabed conditions: (a) flat seabed model and (b) inclined seabed model.

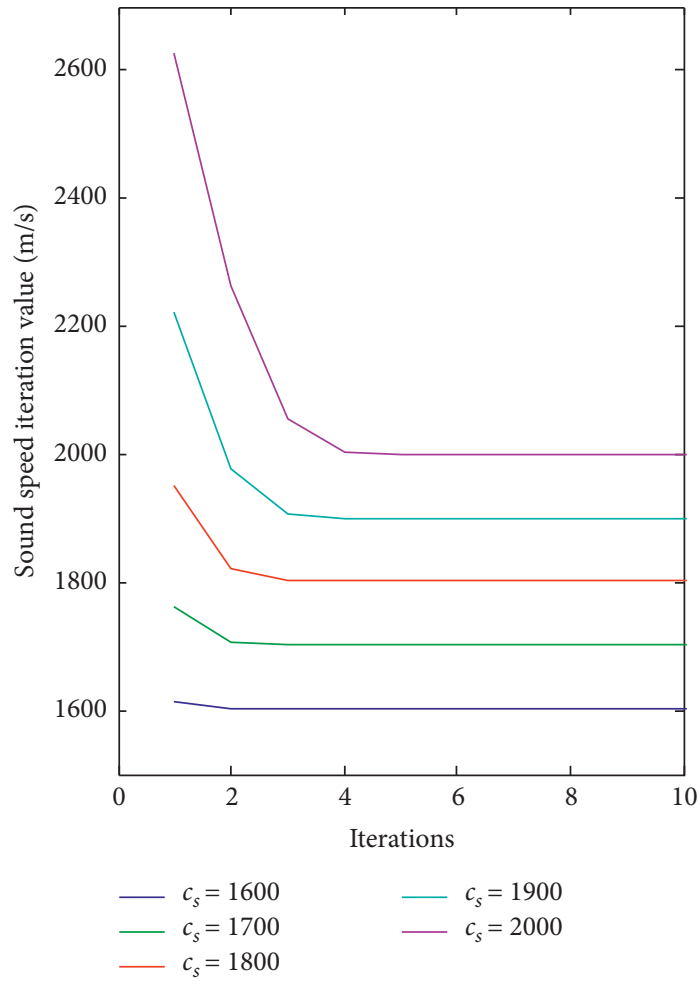

(a)

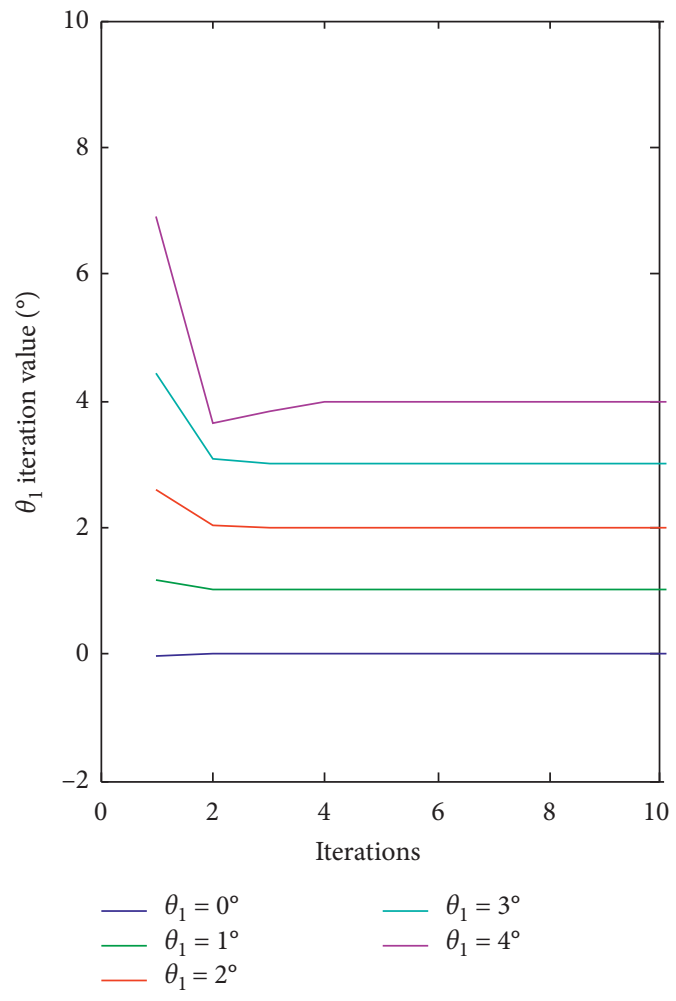

(b)

Figure 3: Convergence rate analysis of Gauss-Newton iteration method under different seabed conditions ( $\operatorname{simulation}$ conditions: $\theta_{0}=10^{\circ}$ ): (a) iterative convergence of sound speed and (b) iterative convergence of $\theta_{1}$. 


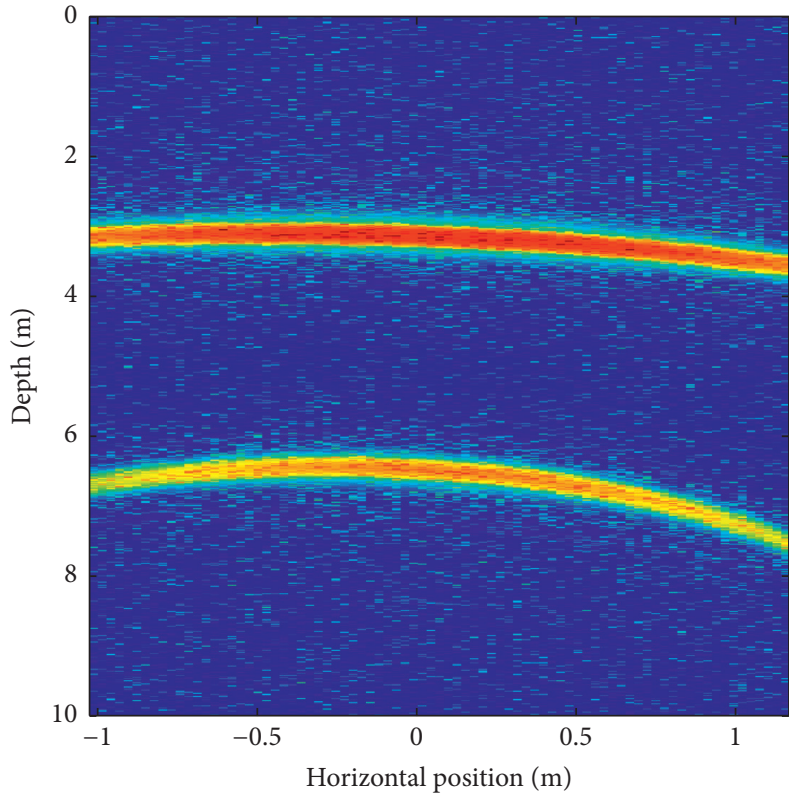

(a)

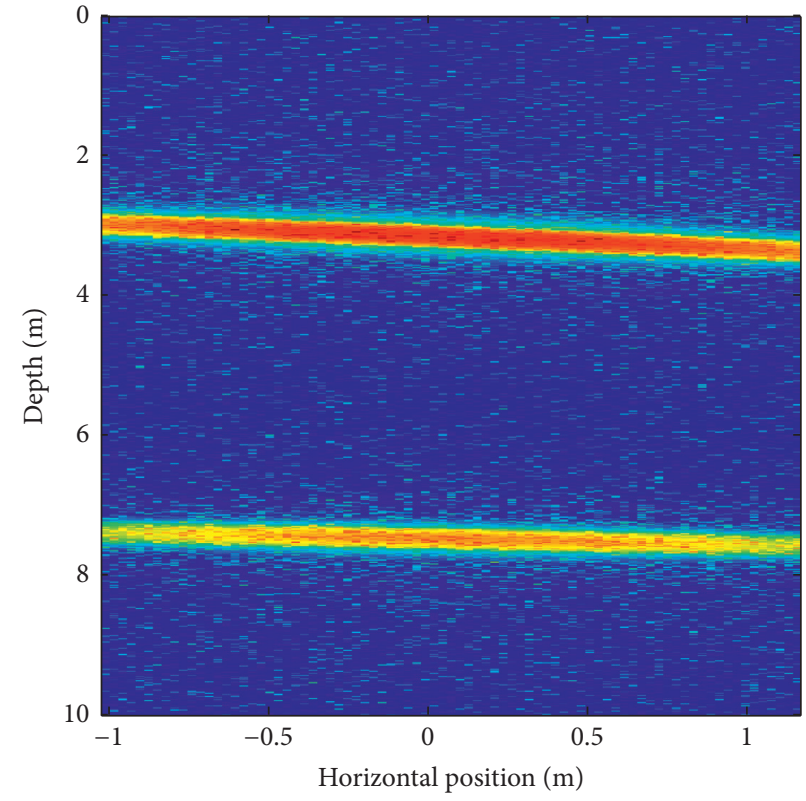

(b)

Figure 4: Phased control sound beams detection of sediment profiles (simulation conditions $\theta_{0}=10^{\circ}, \theta_{1}=5^{\circ}, c_{s}=2000, h=3 \mathrm{~m}$, and $H=4 \mathrm{~m}$ ): (a) before correction and (b) after correction.

sediment. As shown in Figure 1, when the sound beam arrived at the upper sediment layer that corresponds to the sea bottom, the propagation path and the thickness of the sediment conformed to the sine theorem. The thickness of the deposited layer at the seabed footprint is

$$
H_{i}=\frac{1}{2} \frac{\cos \left(\theta_{0}-\theta_{1}\right)}{\sin \left(\beta_{i}+\theta_{1}\right)}\left(T_{i}-t_{i}\right) c_{s}
$$

Figure 4 shows the sub-bottom profiles detected by the phased parametric array by radiating sound beams from $-20^{\circ}$ to $20^{\circ}$ in $0.5^{\circ}$ steps scanning the seabed. The correction results of equations (1) and (16) are shown in Figure 4(b), which solves the problem that the contour of the sediment layer is gradually distorted with the increase of angle and improves the accuracy of sub-bottom profiling.

\section{Conclusions}

An inclined seabed model based on the method of multiangle seabed backscattering signals detected by the phased parametric array is used in sediment sound velocity inversion. Compared with the existing flat seabed model, this model greatly improves the possibility of the acoustic parameters inversion of the actual seabed. This work provides a base work for further real-time inversion of submarine geoacoustic parameters based on the multiangle backscattering signal processing method. In the process of actual shallow stratum profile detection, the accuracy of the sediment profile detected by the phased parametric array sonar system can be corrected by the angle and real-time inversion sound velocity information. Thereby, the model and the method proposed in this article can greatly improve the detection efficiency.

\section{Data Availability}

The data used to support the findings of this study are available from the corresponding author upon request.

\section{Conflicts of Interest}

The authors declare no conflicts of interest.

\section{Acknowledgments}

This work was supported by the NSFC-Zhejiang Joint Fund for the Integration of Industrialization and Informatization (No. U1809212), NSFC-Shandong Joint Fund for Marine Science Research Centers (No. U1906218), the National Natural Science Foundation of China (Nos. 41306182, 41327004, 41606115, and 61401112).

\section{References}

[1] Y.-Y. Zhang, Y. Zhang, G.-L. Hou, and J. C. Sun, “Acoustic intensity flux in low frequency acoustic field of shallow water and its application research," in Proceedings of the 2012 2nd International Conference on Computer Science and Network Technology, pp. 1914-1917, Changchun, China, December 2012.

[2] Y. Zhou and F. Tong, "Research and development of a highly reconfigurable OFDM MODEM for shallow water acoustic communication," IEEE Access, vol. 7, pp. 123569-123582, 2019.

[3] S. Yoshizawa, H. Tanimoto, and T. Saito, "Experimental results of OFDM rake reception for shallow water acoustic communication," in Proceedings of the 2016 Techno-Ocean (Techno-Ocean), pp. 185-188, Kobe, Japan, October 2016. 
[4] Y. Cheng, A. Zhao, J. Hui, T. An, and B. Zhou, "Parametric underwater transmission based on pattern time delay shift coding system," Mathematical Problems in Engineering, vol. 2018, Article ID 8249245, 7 pages, 2018.

[5] G. Theuillon, Y. Stephan, and A. Pacault, "High-resolution geoacoustic characterization of the seafloor using a subbottom profiler in the gulf of lion," IEEE Journal of Oceanic Engineering, vol. 33, no. 3, pp. 240-254, 2008.

[6] M. Gutowski, J. Malgorn, and M. Vardy, "3D sub-bottom profiling-high resolution 3D imaging of shallow subsurface structures and buried objects," in Proceedings of the Oceans 2015-Genova, pp. 1-7, Genoa, Italy, May 2015.

[7] D. Wang, L. Zhang, C. Bao, S. Ma, and Y. Wang, "A comparative study for shallow water match-field inversion using surrogate models," in Proceedings of the Oceans 2019-Marseille, pp. 1-6, Marseille, France, June 2019.

[8] S. E. Dosso and M. J. Wilmut, "Data uncertainty estimation in matched-field geoacoustic inversion," IEEE Journal of Oceanic Engineering, vol. 31, no. 2, pp. 470-479, 2006.

[9] X. Zhu, Z. Wang, and H. Ge, "Matched field processing for a short vertical array in shallow water using the geoacoustic parameters inversed from short range source data," in Proceedings of the 2016 IEEE/OES China Ocean Acoustics (COA), pp. 1-5, Harbin, China, January 2016.

[10] L. Y. S. Chiu, A. Y. Y. Chang, H. Chen, C. Wang, and J. Y. Lou, "Error analysis on normal incidence reflectivity measurement and geoacoustic inversion of ocean surficial sediment," in Proceedings of the 2019 IEEE Underwater Technology (UT), pp. 1-10, Kaohsiung, Taiwan, April 2019.

[11] S. G. Schock, "Remote estimates of physical and acoustic sediment properties in the South China Sea using chirp sonar data and the biot model," IEEE Journal of Oceanic Engineering, vol. 29, no. 4, pp. 1218-1230, 2004.

[12] Y. Kunde, M. Yuanliang, S. Chao, J. H. Miller, and G. R. Potty, "Multistep matched-field inversion for broad-band data from ASIAEX2001," IEEE Journal of Oceanic Engineering, vol. 29, no. 4, pp. 964-972, 2004.

[13] S. G. Schock, "A method for estimating the physical and acoustic properties of the sea bed using chirp sonar data," IEEE Journal of Oceanic Engineering, vol. 29, no. 4, pp. 1200-1217, 2004.

[14] M. R. Fallat, P. L. Nielsen, S. E. Dosso, and M. Siderius, "Geoacoustic characterization of a range-dependent ocean environment using towed array data," IEEE Journal of Oceanic Engineering, vol. 30, no. 1, pp. 198-206, 2005.

[15] T. Zhou, H. Li, J. Zhu, and Y. Wei, “A geoacoustic estimation scheme based on bottom backscatter signals from multiple angles," Acta Physica Sinica, vol. 8, no. 8, pp. 208-214, 2014.

[16] H. Li, J. Ma, J. Zhu, and B. Chen, "Numerical and experimental studies on inclined incidence parametric sound propagation," Shock and Vibration, vol. 2019, Article ID 2984191, 10 pages, 2019.

[17] L. Wan, X. Kong, and F. Xia, "Joint range-Doppler-angle estimation for intelligent tracking of moving aerial targets," IEEE Internet of Things Journal, vol. 5, no. 3, pp. 1625-1636, 2018.

[18] F. Wen, Z. Zhang, K. Wang, G. Sheng, and G. Zhang, “Angle estimation and mutual coupling self-calibration for ULAbased bistatic MIMO radar," Signal Processing, vol. 144, pp. 61-67, 2018.

[19] X. Wang, L. Wang, X. Li, and G. Bi, "Nuclear norm minimization framework for DOA estimation in MIMO radar," Signal Processing, vol. 135, pp. 147-152, 2017.
[20] L. Zhang and T. Zhang, "A robust calibration method for the underwater transponder position based on Gauss-Newton iteration algorithm," in Proceedings of the 2019 7th International Conference on Control, Mechatronics and Automation (ICCMA), pp. 448-453, Delft, Netherlands, November 2019. 